\title{
COMPARAÇÃO DE TIRAS REAGENTES PARA URINÁLISE VETERINÁRIA
}

\author{
Gabriela Fiuza Corato ${ }^{1}$ \\ Ronaldo Eugênio de Oliveira² \\ Kamila Pandolfi ${ }^{3}$ \\ Rudson da Silva Florêncio 4 \\ Mirelle Baptista Jordaim ${ }^{5}$ \\ Leandro Andre Milholli ${ }^{6}$ \\ Filipe Freire Rampinelli ${ }^{7}$ \\ Gabriela Porfirio-Passos ${ }^{8}$ \\ Lenir Cardoso Porfirio ${ }^{9}$
}

\begin{abstract}
Resumo: No exame de urina é possivel identificar parâmetros como $\mathrm{pH}$, glicose, cetona, proteína, bilirrubina, urobilinogênio, densidade, hemoglobina, leucócitos, ácido ascórbico, outros e o exame do sedimento urinário. Este estudo foi desenvolvido para comparar as marcas de tiras reagentes de urina Roche Combur10 Test ${ }^{\circledR}$ UX, Labtest Uriquest Plus VET $T^{\circledR}$ e Inlab Uri-test11 ${ }^{\circledR}$ com a marca utilizada na rotina do Laboratório de Análises Clínicas, a Labtest Uriquest Plus ${ }^{\circledR}$. Foi observada discrepâncias entre os resultados das marcas desenvolvidas para a medicina humana com a marca desenvolvida para a medicina veterinária e entre elas, nos exames da urina de cães atendidos no Hospital Veterinário. Foi possível observar que os parâmetros de $\mathrm{pH}$, densidade e proteína urinária apresentaram maiores discrepâncias entre as fitas quando comparado com padrões para dosagens da densidade por refratometria e da proteína por espectrofotometria. Há necessidade de padronização do exame de urina com tiras reagentes, pois é um valioso auxílio ao diagnóstico de doenças renais e extra-renais.
\end{abstract}

Palavras-chave: Exame químico; Exame de urina; Cães.

\footnotetext{
${ }^{1}$ Medicina Veterinária /Universidade Federal do Espirito Santo Brasil. E-mail: gabrielafiuza@hotmail.com. ${ }^{2}$ Medicina Veterinária /Universidade Federal do Espirito Santo Brasil. E-mail: ronaldoderive11@hotmail.com.

${ }^{3}$ Medicina Veterinária /Universidade Federal do Espirito Santo Brasil. E-mail: kamilapandolfi@yahoo.com.br.

${ }^{4}$ Medicina Veterinária /Universidade Federal do Espirito Santo Brasil. E-mail: rudi.florencio@gmail.com.

${ }^{5}$ Medicina Veterinária /Universidade Federal do Espirito Santo Brasil. E-mail: mirelle_bj@hotmail.com.

${ }^{6}$ Medicina Veterinária /Universidade Federal do Espirito Santo Brasil. E-mail: milholli.leandroandre72@hotmail.com.

${ }^{7}$ Medicina Veterinária /Universidade Federal do Espirito Santo Brasil. E-mail: filipi_upa@gmail.com.

${ }^{8}$ Medicina Veterinária /Universidade Federal da Bahia, Brasil. E-mail: gporfiriopassos@gmail.com.

${ }^{9}$ Medicina Veterinária/Universidade Federal do Espirito Santo Brasil. E-mail: Ienircp52@gmail.com.
} 